\title{
Magnetic viscosity effect in ac susceptibility measurements
}

\author{
I. Klik and Y. D. Yao \\ Institute of Physics, Academia Sinica, Nankang, Taipei, 115 Taiwan \\ X. Yan \\ Department of Physics, Hong Kong University of Science and Technology, Clear Water Bay, Kowloon, Hong Kong, China \\ C. R. Chang \\ Department of Physics, National Taiwan University, Taipei, 107 Taiwan
}

(Received 4 February 1997)

\begin{abstract}
The linear and nonlinear initial ac susceptibilities of thermally relaxing particulate media are derived perturbatively from a master equation. A distribution of activation energies endows the real part of linear susceptibility with a logarithmic dependence on frequency in analogy to magnetic viscosity in dc fields, the proportionality constant is found to be only weakly dependent on temperature via its dependence on thermal stability of the equilibrium state. The temperature and frequency range of this ac viscosity effect are enhanced by ferromagnetic coupling and the predicted behavior is compared with experimental data for $\mathrm{Ni}_{x}\left(\mathrm{SiO}_{2}\right)_{1-x}$ granular films. [S0163-1829(98)00901-1]
\end{abstract}

The theory of thermally activated decay of a metastable state predicts an exponential time dependence while in experiment, as a rule, one detects a decay law far better approximated by the logarithmic function. In magnetic materials this phenomenon is known as the magnetic viscosity effect $^{1-4}$ and since the works of Street and Wolley ${ }^{1}$ it is associated with a distribution of barrier heights within the thermally relaxing sample.

A distribution of barrier heights has an equally dramatic influence on the nonequilibrium initial ac response. A master equation analysis predicts (see below) that the linear ac susceptibility $\chi_{1}^{(1)}(\omega) \propto\left(\omega^{2}+4 \kappa_{0}^{2}\right)^{-1}$, where $\omega$ is the probing field frequency and $\kappa_{0}$ is the thermal relaxation rate. Rather than this inverse power law, however, a logarithmic dependence on frequency, with proportionality constant $S_{\mathrm{ac}}$,

$$
\chi_{1}^{(1)}(\omega) \propto-S_{\mathrm{ac}} \ln \omega,
$$

has recently been reported ${ }^{5}$ for particulate media. We show here that this phenomenon shares a common origin with the magnetic viscosity effect and analyze the temperature and coupling strength dependence of the ac magnetic viscosity $S_{\text {ac }}$ defined by Eq. (1). The theoretical analysis is then compared with experimental data for $\mathrm{Ni}_{x}\left(\mathrm{SiO}_{2}\right)_{1-x}$ granular films.

We consider first a model noninteracting medium formed by an array of single domain, uniaxial ferromagnetic particles with anisotropy constant $K$, activation volume $V$, and energy

$$
E / K V=\sin ^{2}(\theta-\beta)-2 h \cos \theta .
$$

The easy axis and the magnetization vector $\vec{M}$ span, respectively, the angles $\beta$ and $\theta$ with the applied field $H(t)=H_{0} \cos \omega t$; for $|\vec{M}|=M_{s}$ we further define the nucleation field $H_{n}=2 \mathrm{~K} / M_{s}$, the reduced applied field $h=H / H_{n}$ and the reduced projection of magnetization $m=(\vec{M} \cdot \vec{h}) / M_{s}$, into the field direction. At small fields $|h| \ll 1$ the particle has two energy minima whose occupation probabilities $n_{1}$ and $n_{3}=1-n_{1}$ satisfy the master equation

$$
d n_{1} / d t=-\kappa_{13} n_{1}+\kappa_{31} n_{3},
$$

where $\kappa_{i j}$ are thermally activated transition rates defined as

$$
\kappa_{i j}=f_{0} e^{-\left(E_{2}-E_{i}\right) / T}+f_{0} e^{-\left(E_{4}-E_{i}\right) / T},
$$

where $T$ is temperature $\left(k_{B}=1\right)$ and the prefactor is estimated $^{4}$ as $f_{0} \approx 10^{10} \mathrm{~Hz}$. For $h=0$ the minima ( $i=1$ and 3 ) and maxima ( $i=2$ and 4) of Eq. (2) are located at $\theta_{i}=\beta+\pi(i-1) / 2$, their perturbed positions may be determined by solving the equation $d E / d \theta=0$ in separate powers of $h_{0}$. There is

$$
\kappa_{31}(h)=\kappa_{13}(-h)=\sum_{l=0}^{\infty} \kappa_{l} h_{0}^{l} \cos ^{l} \omega t,
$$

where the zero-field relaxation rate $\kappa_{0}=f_{0} e^{-Q / T}, Q=K V$, and after some algebra the higher-order coefficients are found to be $\kappa_{1}=2 q \kappa_{0} \cos \beta, \kappa_{2}=q(2 q-1) \kappa_{0}$, and

$$
\kappa_{3}=(q / 6)\left[16 q^{2}-12 q-3-\left(8 q^{2}-3\right) \cos 2 \beta\right] \kappa_{0} \cos \beta,
$$

with $q=Q / T$. The steady-state, particular solutions of Eq. (3) then lead to ${ }^{6}$

$$
\begin{aligned}
m(t) & =n_{1} \cos \theta_{1}+n_{3} \cos \theta_{3} \\
& =\sum_{\text {odd } l}\left[\chi_{l}^{(1)} \cos l \omega t+\chi_{l}^{(2)} \sin l \omega t\right] h_{0}^{l},
\end{aligned}
$$

and the two lowest-order susceptibility components are

$$
\chi_{1}^{(j)}=\delta_{1}^{(j)} \sin ^{2} \beta+2 X_{1}^{(j)} \cos \beta,
$$


$\chi_{3}^{(j)}=\frac{1}{2} \delta_{1}^{(j)} \sin ^{2} \beta \cos ^{2} \beta+\frac{3}{4} X_{1}^{(j)} \cos \beta \sin ^{2} \beta+2 X_{3}^{(j)} \cos \beta$,

( $\delta_{i}^{(j)}=1$ if $j=i$ and zero otherwise) in their respective leading orders of $h_{0}$. The temperature independent terms $\left(\sim \delta_{i}^{(j)}\right)$ are due to reversible shifts of the energy minima in Eq. (2) and the remaining terms, due to thermally activated magnetization reversals, contain the coefficients $X_{2 i+1}^{(j)}$ obtained from perturbative solutions of Eq. (3):

$$
\begin{gathered}
\Delta_{1}^{2} X_{1}^{(1)}=2 \kappa_{0} \kappa_{1}, \\
\Delta_{1}^{2} X_{1}^{(2)}=\kappa_{1} \omega, \\
2 \Delta_{1}^{2} \Delta_{3}^{2} X_{3}^{(1)}=\omega^{2}\left(\kappa_{0} \kappa_{3}+\kappa_{1} \kappa_{2}\right)+4 \kappa_{0}^{2}\left(\kappa_{0} \kappa_{3}-\kappa_{1} \kappa_{2}\right), \\
4 \Delta_{1}^{2} \Delta_{3}^{2} X_{3}^{(2)}=\omega\left[3 \omega^{2} \kappa_{3}+4 \kappa_{0}\left(3 \kappa_{0} \kappa_{3}-4 \kappa_{1} \kappa_{2}\right)\right],
\end{gathered}
$$

$\Delta_{l}^{2}=(l \omega)^{2}+4 \kappa_{0}^{2}$. The nonequilibrium linear ac susceptibility of Eq. (6) is well known, but Eq. (7) for the nonlinear ac response appears to be new, complementing the previously published results for susceptibility of free rotators ${ }^{6,7}$ and Ising magnets. ${ }^{8}$

For typical media the susceptibilities (6) and (7) are to be averaged over a uniform distribution of the deviation angle $\beta$ and over a distribution of the barrier heights $Q$. The averages over $\beta$ follow from the formula $\cos ^{2 n} \theta=1 /(2 n+1)$ and for definiteness we take here the log-normal ${ }^{1-5}$ distribution of $Q$ :

$$
P(Q)=(2 \pi)^{-1 / 2}(\sigma Q)^{-1} \exp \left[-\ln ^{2}\left(Q / Q_{0}\right) /\left(2 \sigma^{2}\right)\right] .
$$

However, rather than considering directly the $Q$ averages of Eqs. (6) and (7) we invoke a geometric argument familiar from studies of magnetic viscosity ${ }^{4}$ and address first the derivatives

$$
-\left\langle\frac{\partial \bar{\chi}_{1}^{(j)}}{\partial \ln \omega}\right\rangle=\frac{4}{3 \sigma \sqrt{2 \pi}} \frac{Q_{0}}{T} \int_{0}^{\infty} d x p(x) g_{1}^{(j)}(x),
$$

$x=Q / Q_{0}$. The function $p(x)=\exp \left[-\ln ^{2} x /\left(2 \sigma^{2}\right)\right]$ originates from the distribution (12) and the functions

$$
\begin{aligned}
& \widetilde{\Delta}_{1}^{4}(x) g_{1}^{(1)}(x)=4 \omega^{2} \widetilde{\kappa}_{0}^{2}(x), \\
& \widetilde{\Delta}_{1}^{4}(x) g_{1}^{(2)}(x)=\omega \widetilde{\kappa}_{0}(x)\left[\omega^{2}-4 \widetilde{\kappa}_{0}^{2}(x)\right],
\end{aligned}
$$

follow from Eqs. (6) and (8), with $\widetilde{\kappa}_{0}(x)=f_{0} e^{-q_{0} x}$, $q_{0}=Q_{0} / T$ and $\widetilde{\Delta_{l}^{2}}(x)=(l \omega)^{2}+4 \widetilde{\kappa_{0}^{2}}(x)$.

The integrand in Eq. (13) is a product of the two functions $p(x)$ and $g_{1}^{(j)}(x)$. The function $p(x)$ peaks around $x_{0}=1$, while $g_{1}^{(1)}(x)$ is sharply localized around the point

$$
x_{1}^{\prime}=\frac{T}{Q_{0}} \ln \frac{2 f_{0}}{\omega},
$$

i.e., for $2 \widetilde{\kappa}_{0}\left(x_{1}^{\prime}\right)=\omega$, with peak value $g_{1}^{(1)}\left(x_{1}^{\prime}\right)=1 / 4$. The logarithmically slow shift of $x_{1}^{\prime}$ along the $x$ axis ensures that

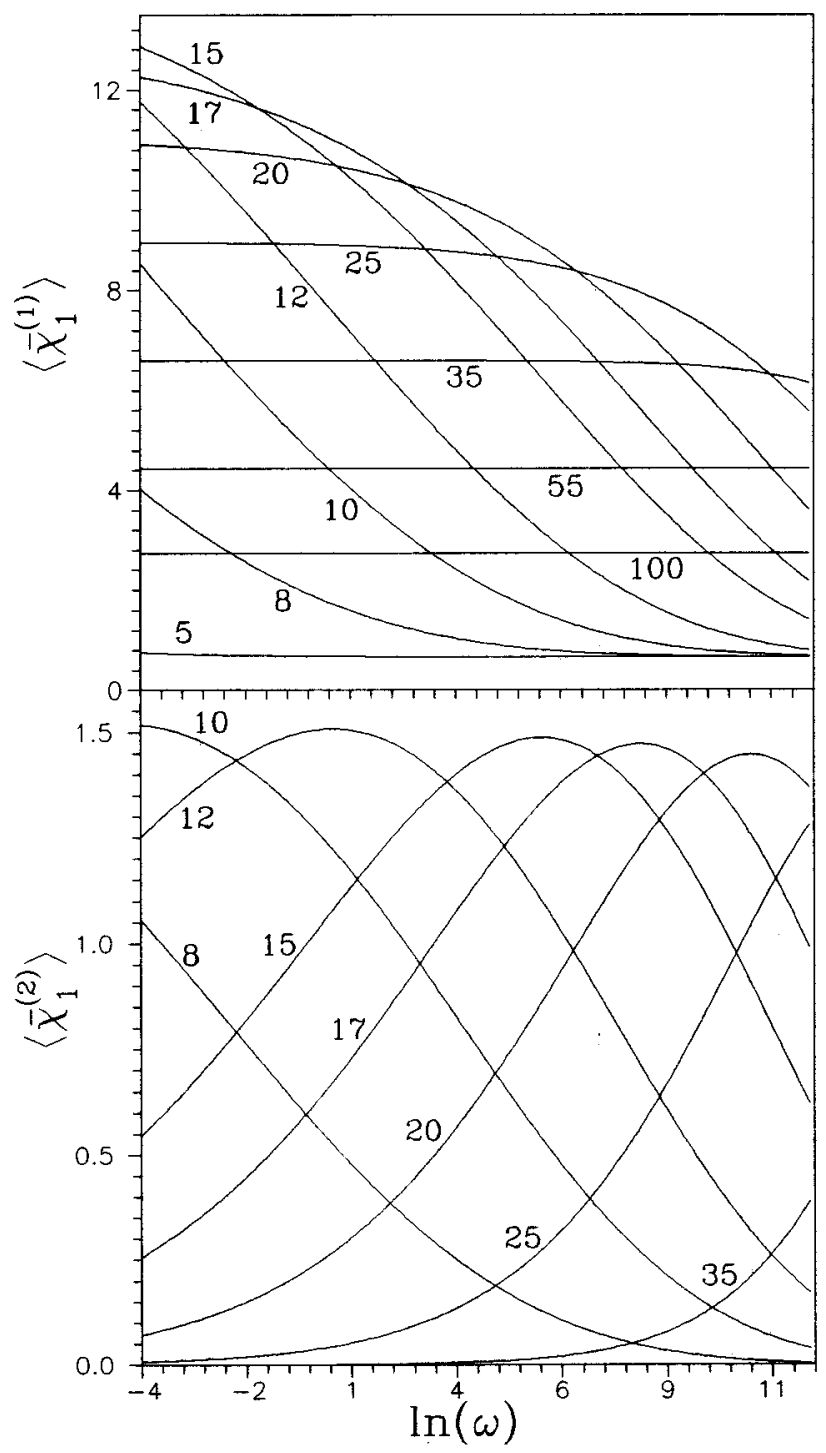

FIG. 1. The two components of the linear ac susceptibility given by Eq. (6) averaged over $Q$ and $\beta$. Model parameters: $Q_{0}=300 \mathrm{~K}$, $\sigma=0.27$ and the prefactor $f_{0}=e^{25} \mathrm{~Hz}$. Curve labels denote temperature in $\mathrm{K}$.

for sufficiently large $\sigma$ the two peaks overlap over a large range of frequencies $\omega$ and in this overlap range the integral in Eq. (13) maintains its maximum, $\omega$-independent value, in full analogy to slow magnetization decay in constant applied field. ${ }^{4}$ To every temperature $T$, barrier height $Q_{0}$, and distribution width $\sigma$ there corresponds therefore a specific range of frequencies in which the linear ac susceptibility $\left\langle\bar{\chi}_{1}^{(1)}(\omega)\right\rangle$ exhibits logarithmic $\omega$ dependence. Moreover, within the overlap range the ac magnetic viscosity $S_{\text {ac }}$ does not depend on $T$ since on setting $p(x) \equiv 1$ in Eq. (13) we obtain

$$
3 \sigma \sqrt{2 \pi} S_{\mathrm{ac}} \approx 2 \frac{4 f_{0}^{2}}{4 f_{0}^{2}+\omega^{2}} \approx 2
$$

for $\omega \ll f_{0}$. This behavior is apparent from Fig. 1 where we plot the averaged linear susceptibilities $\left\langle\bar{\chi}_{1}^{(j)}(\omega)\right\rangle$ versus $\ln \omega$. At medium temperatures the slope of $\left\langle\bar{\chi}_{1}^{(j)}(\omega)\right\rangle$ is ap- 


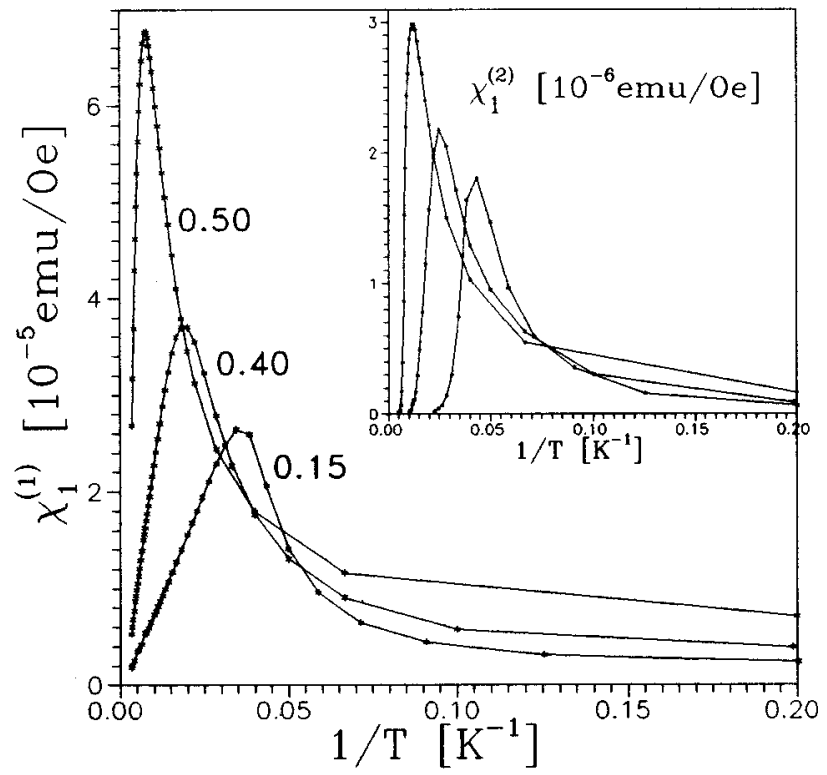

FIG. 2. Measured linear susceptibility $\chi_{1}^{(1)}$ versus inverse temperature for $\mathrm{Ni}_{x}\left(\mathrm{SiO}_{2}\right)_{1-x}$ granular films. Nominal nickel volume fraction $x$ as labeled and probing field frequency $f=\omega / 2 \pi=1 \mathrm{~Hz}$. All samples are the same size. Inset shows the corresponding curves for the dissipative part $\chi_{1}^{(2)}$.

proximately constant, while at very low and very high $T$, where there is no overlap of $p(x)$ and $g_{1}^{(1)}(x)$, the slope is zero.

The dissipative linear response $\left\langle\bar{\chi}_{1}^{(2)}(\omega)\right\rangle$ is amenable to similar analysis: The function $g_{1}^{(2)}(x)$ of Eq. (15) vanishes identically at $x_{1}^{\prime}$ and reaches a minimum for $2 \xi_{1} \widetilde{\kappa}_{0}\left(x_{1,1}^{\prime \prime}\right)=\omega$ and a maximum for $2 \xi_{2} \widetilde{\kappa}_{0}\left(x_{1,2}^{\prime \prime}\right)=\omega$ where $\xi_{i}^{2}=3$ $+(-1)^{i} 2 \sqrt{2}$. There is $x_{1,1}^{\prime \prime}<x_{1}^{\prime}<x_{1,2}^{\prime \prime}$, and the peak values $g_{1}^{(2)}\left(x_{1, i}^{\prime \prime}\right)=(-1)^{i} / 8$. The two extrema shift logarithmically slowly along the $x$ axis and the sign and magnitude of the integral (13) depend on the overlap of $p(x)$ and $g_{1}^{(2)}(x)$. In particular, if $p(x)$ overlaps both peaks of $g_{1}^{(2)}(x)$ then the integral (13) is approximately zero and $\left\langle\vec{\chi}_{1}^{(2)}(\omega)\right\rangle$ attains its maximum value within the validity range of Eq. (1). In the present noniteracting model the peak value of $\left\langle\bar{\chi}_{1}^{(2)}(\omega)\right\rangle$ is independent of temperature (see also Fig. 1) since for $\omega \ll f_{0}$

$$
3 \sigma \sqrt{2 \pi} \max _{\omega}\left\langle\bar{\chi}_{1}^{(2)}(\omega)\right\rangle \approx \pi
$$

It is also possible to define the functions $g_{3}^{(j)}(x)$ corresponding to the averaged nonlinear susceptibilities $\left\langle\bar{\chi}_{3}^{(j)}(\omega)\right\rangle$. These functions exhibit essentially the same behavior as $g_{1}^{(j)}(x)$, with the important difference that not only the peak positions, but also their heights vary with $\ln \omega$ and plots of $\left\langle\bar{\chi}_{3}^{(1)}(\omega)\right\rangle$ (not shown) illustrate the concomitant loss of logarithmic $\omega$ dependence. By the same token, the function $\max _{\omega}\left\langle\bar{\chi}_{3}^{(2)}(\omega)\right\rangle$ varies approximately as $T^{-2}$. The functions $\left\langle\vec{\chi}_{3}^{(j)}(\omega)\right\rangle$ are negative, about two orders of magnitude larger (in absolute value) than $\left\langle\bar{\chi}_{1}^{(j)}(\omega)\right\rangle$ so that the first two terms in Eq. (7) may be neglected.

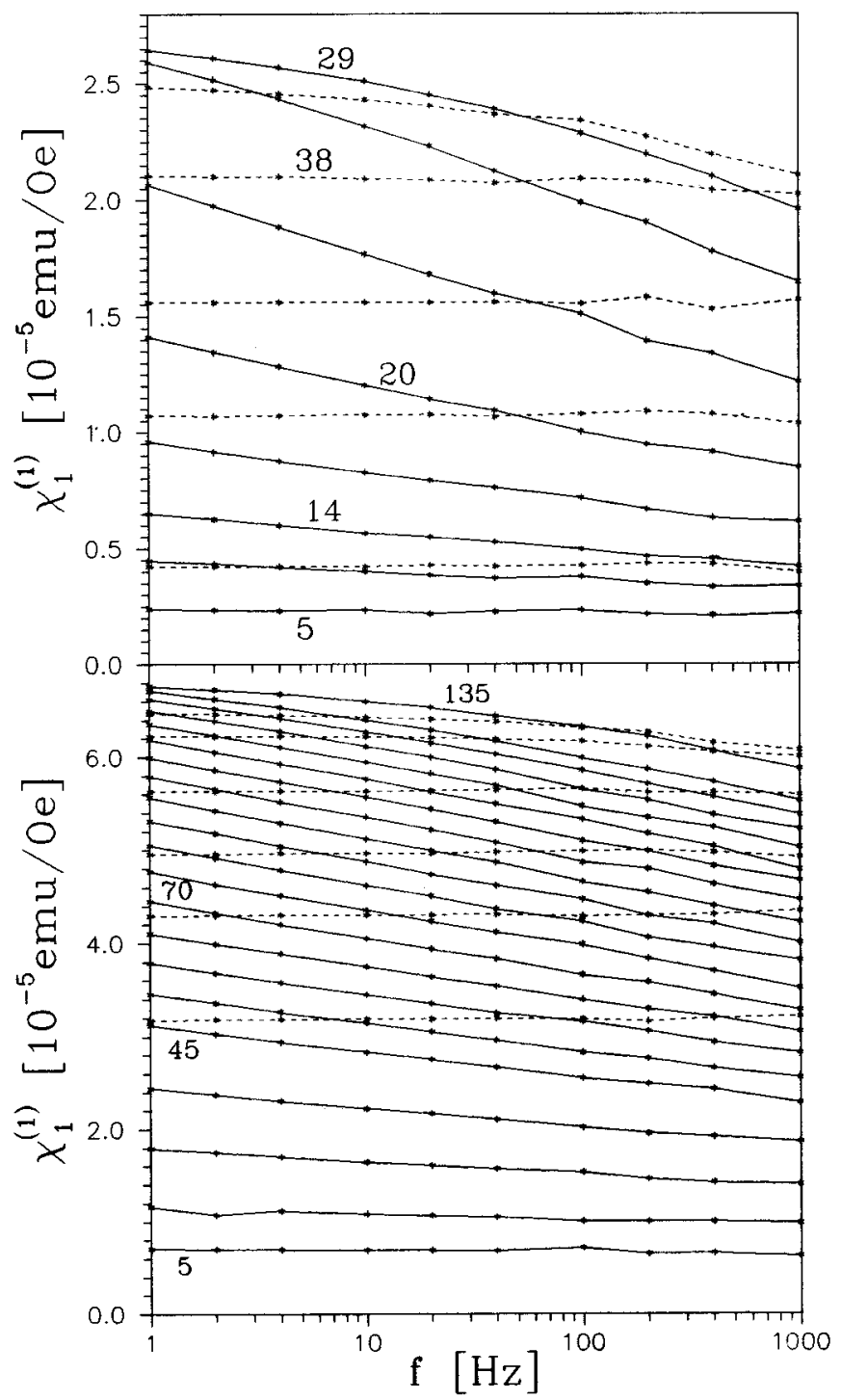

FIG. 3. Measured linear susceptibility $\chi_{1}^{(1)}$ versus frequency at selected values of temperature for $\mathrm{Ni}_{x}\left(\mathrm{SiO}_{2}\right)_{1-x}$ granular films. The $f=1 \mathrm{~Hz}$ data correspond to Fig. 3. Above: $x=0.15$ and temperature $T=5,11,14, \ldots, 29,32,38,50,70$, and $160 \mathrm{~K}$. For $T>29 \mathrm{~K}$ the curves are shown in dashed lines. Below: $x=0.50$ and temperature $T=5,15,25,35,45,50, \ldots, 115,120,135,155,165,185,205$, 225 , and $265 \mathrm{~K}$. Selected curves in both plots are labeled by $T$.

The relation of the activation volume $V$ to the saturation magnetic moment $\mathcal{M}_{s}$ is unclear ${ }^{9}$ and in writing down the average (13) we assumed that all particles contribute the same moment. Our analysis, however, holds also in the contrary case of $\mathcal{M}_{s} \sim M_{s} V$ where $p(x)=x \exp \left[\sigma^{2}-\ln ^{2} x /\left(2 \sigma^{2}\right)\right]$ with a local maximum at $\ln x_{0}=\sigma^{2}$ and $p\left(x_{0}\right)=1$. The relation of $\mathcal{M}_{s}$ and $V$ is therefore not an essential factor ${ }^{4}$ in determining the existence and range of the ac magnetic viscosity effect which, as we show below, depend far more strongly on interparticle interactions.

As an exactly solvable example of an interacting system we consider a particle pair with dipolar coupling ${ }^{10}$ and bond angles $\theta_{b}=0$ and $\pi / 2$ corresponding to ferromagnetic (FM) and antiferromagnetic (AFM) mutual ordering, respectively. The particles are identical, with easy axes parallel to the external field. The evolution of the bound pair is described 
by a three-level master equation ${ }^{10}$ whose perturbative solutions yield

$$
\begin{aligned}
& \left(\kappa_{0}+\gamma_{0}\right) \Delta_{1}^{2} \chi_{1}^{(1)}=16 q \kappa_{0}^{2} \gamma_{0}, \\
& \left(\kappa_{0}+\gamma_{0}\right) \Delta_{1}^{2} \chi_{1}^{(2)}=8 q \omega \kappa_{0}^{2} \gamma_{0},
\end{aligned}
$$

with decay rates $\kappa_{0}=f_{0} \exp \left[-q\left(h_{n}^{2}+\varepsilon \rho\right)\right]$ and $\gamma_{0}=f_{0} \exp \left[-q h_{n}^{2}\right]$, frequency $\Delta_{1}^{2}=\omega^{2}+4 \kappa_{0}^{2}$ and critical field ${ }^{10} h_{n}^{2}=(1-\rho)(1 \pm 3 \rho)$ if $\theta_{b}=0$ (upper sign) and $\pi / 2$ (lower sign). The parameter $\varepsilon=8$ for FM and -4 for AFM coupling and the dimensionless dipolar coupling strength $\rho$ between two particles a distance $R$ apart is $\rho=M_{s}^{2} V / 2 K R^{3}$.

The integrand of the resultant derivative $\left\langle\partial \chi_{1}^{(1)} / \partial \ln \omega\right\rangle$ is again a product of the distribution $p(x)$ and of a sharply peaked function with a maximum at

$$
x_{1}^{\prime}(\rho) \approx \frac{T}{Q_{0}\left(h_{n}^{2}+\varepsilon \rho\right)} \ln \frac{2 f_{0}}{\omega} .
$$

FM coupling destabilizes the demagnetized state, enhances initial susceptibility and shifts the peak of $\chi_{1}^{(1)}$ to higher temperatures. Moreover, in Eq. (21) there is

$$
h_{n}^{2}+\varepsilon \rho= \begin{cases}(1+\rho)(1+3 \rho) & \text { if } \theta_{b}=0 \\ (1+\rho)(1-3 \rho) & \text { if } \theta_{b}=\pi / 2,\end{cases}
$$

so that FM coupling $\left(\theta_{b}=0\right)$ also slows down the shift of $x_{1}^{\prime}(\rho)$ along the $x$ axis and broadens therefore the overlap range where $\left\langle\chi_{1}^{(1)}(\omega)\right\rangle$ has the logarithmic $\omega$ dependence given by Eq. (1). AFM coupling $\left(\theta_{b}=\pi / 2\right)$ has the opposite effect.

We conclude this work by comparing the predicted range of ac magnetic viscosity effect with experimental data for $\mathrm{Ni}_{x}\left(\mathrm{SiO}_{2}\right)_{1-x}$ granular films. The experimental procedure may be found in Refs. 5,11, here we present merely the observed linear ac susceptibility as a function of temperature (Fig. 2) and frequency (Fig. 3). According to Fig. 2 the peak of $\chi_{1}^{(1)}$ shifts to higher temperatures with increasing nominal nickel volume fraction $x$. This shift may be associated with increasing particle size ${ }^{11}$ which, however, should theoretically also decraese the peak height and we find that even for $x=0.15$ the experimental curves cannot be fitted by Eq. (6) for noninteracting particulate arrays. We therefore conclude that the observed enhancement of $\chi_{1}^{(1)}$ is due to FM interactions (see also Ref. 12) described qualitatively by Eq. (19). The temperature range in which the measured linear susceptibility satisfies the logarithmic law (1) increases with the $\mathrm{Ni}$ volume fraction $x$ : For $x=0.15$ (see Fig. 3, top) only curves measured at 20,23 , and $26 \mathrm{~K}$ maintain, approximately, the maximum slope throughout the measured frequency domain, while for $x=0.50$ (Fig. 3, below) the temperature range of the ac viscosity effect extends from 45 to about $120 \mathrm{~K}$, in full agreement with the qualitative prediction of Eq. (21). For coupled systems it is not possible to write down an explicit expression for $S_{\mathrm{ac}}$ similar to Eq. (17), there is, however,

$$
\sigma S_{\mathrm{ac}} \propto\left(h_{n}^{2}+\varepsilon \rho\right)^{-1},
$$

if $\varepsilon>0$ (FM coupling), so that the theoretical value of $S_{\text {ac }}$ decreases with increasing FM coupling strength in accordance with the experimental plots of Fig. 3.

In summary, we find that the here defined ac magnetic viscosity $S_{\mathrm{ac}}$ is a material parameter characterizing the sample composition. Ferromagnetic coupling increases the temperature and frequency range over which the ac viscosity effect may be observed and, at the same time, decreases the value of $S_{\text {ac }}$. Antiferromagnetic coupling has the opposite effect.
${ }^{1}$ R. Street and J. C. Wolley, Proc. Phys. Soc. London, Sect. A 62, 562 (1949); Proc. Phys. Soc. London, Sect. B 55, 679 (1952); 69, 1189 (1956).

${ }^{2}$ P. Gaunt, Philos. Mag. 34, 775 (1976); J. Appl. Phys. 59, 4129 (1986); B. Barbara and L. Gunther, J. Magn. Magn. Mater. 128, 35 (1993).

${ }^{3}$ M. El-Hilo, K. O. Grady, and R. W. Chantrell, J. Magn. Magn. Mater. 109, L164 (1992); A. Labarta, O. Iglesias, Ll. Balcells, and F. Badia, Phys. Rev. B 48, 10240 (1993).

${ }^{4}$ I. Klik and C. R. Chang, Phys. Rev. B 47, 9091 (1993); J. Magn. Magn. Mater. 128, L17 (1993); I. Klik, C. R. Chang, and J. Lee, J. Appl. Phys. 75, 5505 (1994).

${ }^{5}$ B. Zhao, J. Y. Chow, and X. Yan, J. Appl. Phys. 79, 6022 (1996).

${ }^{6}$ T. Bitoh, K. Ohba, M. Takamatsu, T. Shirane, and S. Chikazawa,
J. Phys. Soc. Jpn. 64, 1311 (1995).

${ }^{7}$ Y. Kimura and R. Hayakawa, Jpn. J. Appl. Phys., Part 1 31, 3387 (1992).

${ }^{8}$ J. L. García and J. Lázaro (unpublished).

${ }^{9}$ A. M. De Witte, K. O'Grady, G. N. Coverdale, and R. W. Chantrell, J. Magn. Magn. Mater. 88, 183 (1990); J. F. Liu and H. L. Luo, ibid. 94, 43 (1991).

${ }^{10}$ W. Chen, S. Zhang, and H. N. Bertram, J. Appl. Phys. 71, 5579 (1992); I. Klik, J. S. Yang, and C. R. Chang, ibid. 76, 6493 (1994); I. Klik and C. R. Chang, Phys. Rev. B 52, 3540 (1995).

${ }^{11}$ Y. Xu, B. Zhao, and X. Yan, J. Appl. Phys. 79, 6137 (1996).

${ }^{12}$ J. I. Gittleman, Y. Goldstein, and S. Bozovski, Phys. Rev. B 5, 3609 (1972). 